\title{
A Contribution to Workplace Ergonomics Evaluation Using Multimedia Tools and Virtual Reality
}

\author{
Roman Leskovský, Erik Kučera, Oto Haffner, Jakub Matišák, Danica Rosinová and Erich Stark \\ Faculty of Electrical Engineering and Information Technology \\ Slovak University of Technology in Bratislava \\ Bratislava, Slovakia \\ Email: roman.leskovsky@stuba.sk, erik.kucera@stuba.sk
}

\begin{abstract}
The paper demonstrates an application developed to help to evaluate ergonomics of a workplace. Ergonomics of a workplace has enormous impact on employees and their longterm work effectiveness, which causes an interest in this field from employers' point of view. The paper describes and compares several attitudes companies use to set up and evaluate workplace metrics, potential of virtual reality (VR) in the process, VR application proposal, implementation within Unity 3D engine and results achieved with implementation of this proposed solution. Current approaches also include motion tracking for ergonomics evaluation. These technologies are often far over smaller companies' budget. Described solution is reasonably priced also for small companies, using cheaper motion capture equipment.
\end{abstract}

\section{INTRODUCTION}

A $\mathrm{T}$ THE present time, the interest in health of employees performing monotonous and repetitive routines in industrial companies is increasingly being pursued. One of the new issues is the evaluation of workplace ergonomics in such enterprises. This topic is strongly supported, especially in Germany, where trade unions have an important influence. The paper deals with the proposal of methodology for ergonomics evaluation using modern information-communication technologies (ICT), such as virtual or mixed reality. This vision is fully in line with the European industry revolution - Industry 4.0 [1].

The aim of our work was to contribute to the development of a comprehensive system (application) for the evaluation of workplace ergonomics for medium and small enterprises. As the development of such a system (application) is a complex task that requires a multidisciplinary approach, the work has set out subtasks that relate to computer support for ergonomics evaluation. The project uses a new motion capture suit named Perception Neuron. The advantage of this solution is a system that is less expensive than current solutions. Another benefit is the use of a virtual reality in which individual workplaces can be composed and, if necessary, used for worker training [2].

\section{ERGONOMICS}

Ergonomics is the process of designing and deployment of workplaces - objects and systems in an environment so that their layout fits the people who use them. It can be applied to all processes and locations that include people - workplaces, sports, leisure activities, but also safety and health.

The goal of ergonomics is to refine the layout of environment to minimize the risk of injury or other types of health problems.

Enterprises are concerned with ergonomics because they realize that the better the environment is adapted to the needs of the person, the higher the productivity of person's work is. At the same time, companies try to minimize the costs of illnesses and wounds resulting associated with work at the workplace.

\section{A. Ergonomics Evaluation}

Several disciplines are used to evaluate ergonomics [3], [4]. In the factories, especially in our site, the implementation is predominantly tabulated, with the company prescribing workplace deployment standards, which describe the minimum / maximum table height, desktop size, distance between objects, and so on.

Some of the companies also use innovative technological solutions that are available today. Motion capture methods have been used at the sport sector. Similarly, there are car manufacturers that evaluate the cockpit ergonomics with the aforementioned methods, which record, for example, the movements of people during boarding and disembarking, which are later evaluated.

We attempt to contribute in this area by incorporating current technologies, using motion capture methods in combination with virtual reality [5].

\section{B. Existing Solutions}

The paper [6] lists the basic ergonomic aspects of workplace design. The advantages of the ergonomic design of the working environment are proven by increasing work performance and reducing errors. A turnover of staff is also reduced. Workers are more satisfied at work and have no reason to look for a new job. The benefits can therefore be seen for both organizing the production and the employee:

1) For the production organization 
a) reduction of incapacity for work and occupational diseases

b) performance improvement

c) reducing error and confusion

d) improving the mental status of the worker

2) For the worker:

a) improving the mental and physical condition of the worker

b) minimizing the signs of mental and physical fatigue

c) social benefits - improved self-realization

As stated in [7], the use of virtual reality technology in solutions aimed at increasing workplace ergonomics is an unavoidable trend. Virtual reality applications have these benefits:

- user can enter and walk through the scene on different tracks

- events happen in real time

- scene and objects have 3D character

- scene is not static and objects can be manipulated

Virtual reality models allow:

- replacing physical prototypes with virtual ones

- simulation of the different stages of development in a virtual environment

- improvement and acceleration of product development processes

- scene is not static and objects can be manipulated

The main advantages of human simulation in the $3 \mathrm{D}$ environment are:

- shortening development time

- reducing development costs

- improvement of quality and safety

- increase of competitiveness

The use of virtual reality in the design of workplace ergonomics will provide the following functionalities:

- a clearer design of the new work cell

- evaluation of existing assembly line, increasing its efficiency considering the human factor

- energy expenditure for the operation

Tecnomatix Jack is frequently used for ergonomic workplace design [8]. Though, it does not provide a built-in solution that uses VR and its unquestionable benefits. Existing solutions for employee ergonomics analysis in VR are mostly developed by big companies. These software and hardware solutions are often developed for automakers [9].

So far, there is no affordable solution for small and mediumsized companies. For example, these are commercially available virtual reality headsets and affordable motion capture suits. Our proposed solution described in this paper is priced at a maximum of thousands of USD / USD. Existing solutions for large companies are priced at hundreds of thousands.

\section{PROPOSED VISION}

The impulse for the project was the idea of connecting motion capture methodology with the visualization capabilities

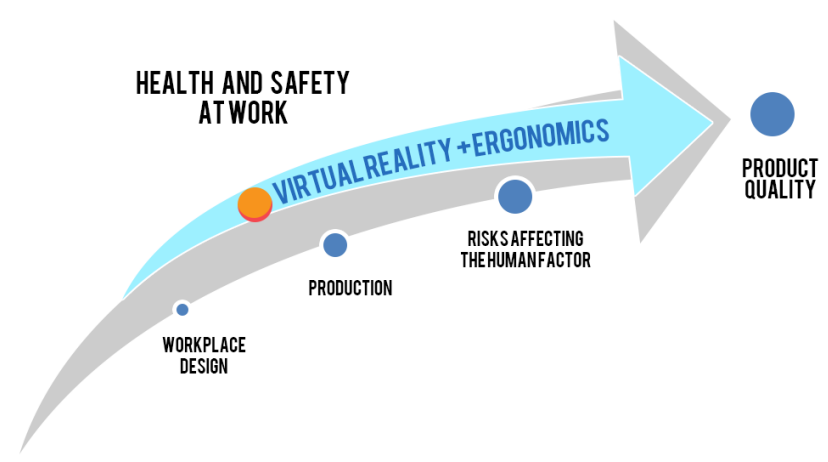

Fig. 1. Impact of involving innovative procedures to enhance product reliability [7]

of VR applications [10] [11], giving users new perspective to the spatial body movement in a virtual workplace.

Recording worker's movements via motion capture technology and then providing users with tools for controlling recorded live movement data similarly as a movie player with functions to control play speed or pause whole moment and also providing tools for modifying environments in way of building custom workplaces, would granted users with full control of those aspects which would make it easy to recognize critical spots of the workplace.

Application use is intended mainly for those kinds of workplaces, where inappropriate ergonomics may cause an increase of wounds. There are connected with manual repetitive actions, that are common e.g. in factories, so it is compulsory that it includes industrial equipment, that could be used to build industrial workplaces. Considering the fact that application should be reasonably priced also for small companies, proper support of inexpensive variants of technologies is required.

\section{Main Aspects Of Proposed Application}

Based on the vision, our application was proposed. The proposal was divided into several different independent parts, each dealing with different problematics. Application was divided into:

- Supported technologies

- User input / movement

- Environment

- User interface

- Workplace building

- Work simulation

- Limitations

\section{A. Supported Technologies}

As it was stated in the proposed vision, the application should offer virtual reality environment, which includes the use of a VR headset. It relies on use of a motion capture technology and 3D engine which is a powerful environment when dealing with $3 \mathrm{D}$ rendering [12]. 
There are a lot of VR headsets to choose from. Support for more than one device often meant device dependent development and writing code for each device separately. It was caused by differences in controllers and input. Some of the headsets provides only gestures, others one or two controllers of different types.

Acer WMR (Windows Mixed Reality) was the best candidate for the application and for testing of the proposed system. Its system requirements and performance tests were better on our computers than it was with the other virtual reality headsets considered for use (including HTC Vive, Oculus Rift). Advantages of this headset is lack of external sensors for tracking of headset's orientation. Tracking sensors are internal - in a form of two cameras detecting movement of the device relative to the room or other surroundings by the changes of positions of referral points in the environment in each image. In addition to performance and saving one HDMI port and not using external sensors, also the price is lower than the price of the other devices. Headset belongs into the family of Windows Mixed Reality headsets. These devices share uniform controllers, which makes the application compatible with each type of headset of this type.

For motion capture technology, price was the key feature that matters as this could be the most expensive part of the whole project. Also used tracking technology was important as motion should be captured right in and during real work process. Some of the workplaces could provide only very constrained setting, making it impossible to capture movement with cameras detecting markers on worker.

Considering mentioned facts, Perception Neuron was selected. Developed by Noitom, this motion capture suit consists of individual sensors called Neurons. Each neuron houses an Inertial Measurement Unit with a accelerometer, gyroscope and magnetometer [13]. This units should provide decent measurement values with reasonable price. Suit allows full body tracking. Noitom also provides a SW in the form of environment, where editing, real-time preview of animations and export to various formats are possible. The only limitations are warnings about wearing suit near the electric devices, which is not always possible in a factory.

\section{B. User Input and Movement}

A design of user input was not an easy task. It is always important to realize, what are the actions that we need to cover and how many different types and keys will be available for the users.

Using WMR controllers, we got four buttons (one reserved for return to Windows), one joystick and one touchpad per controller. Considering left and right hand-oriented users, it may cause difficulties, if each of the buttons on the left and right controller had different functions. It would be also not possible to completely control the application with just one controller. Because of this, functions of buttons on both controllers will be mirrored. This left only five input keys for genuine actions as buttons on the left controller should call the same actions as the opposite buttons on the right controller.
As defined in the vision, main actions could consist of:

- movement

- displaying/hiding the menu

- selecting items in menu / environment

- confirming action

- playing the simulation

- changing simulation play speed

Reserving joystick for movement works great and feels natural as axis of joystick movement corresponds to the direction of movement in a virtual environment. Touchpad was reserved for scrolling in menus on vertical axis and changing play speed on horizontal axis.

As a specific method of location input, application will use controllers as pointers used to select options in menu panels or position in environment.

All buttons setup proposal is described in Fig. 2.

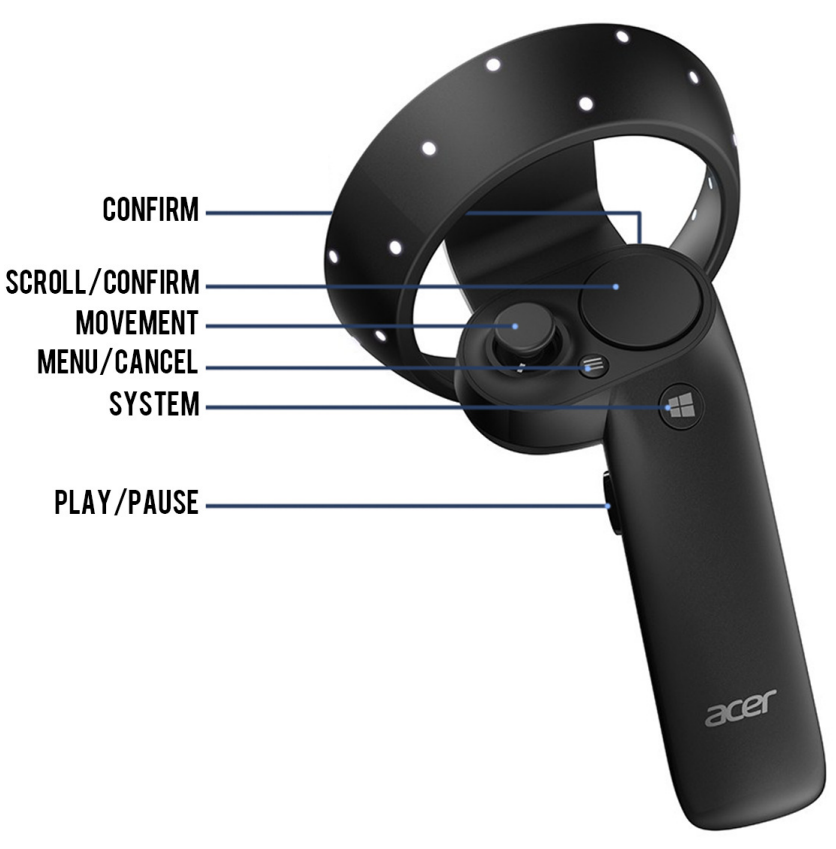

Fig. 2. Controller key bindings

Movement itself was a problem since of appearance of first virtual reality applications. Movement caused disorientation and nausea. Now we know, that this was caused by unnatural movements in virtual environment that human brain had problem to process. Most of the problems were caused by absenting acceleration and deceleration forces present while moving in real life. This would be impossible to do by code, as every person's movement pattern is different from others. Therefore, modern VR applications relies on real physical movement that is detected through inside or outside sensors and then it is transferred into the VR, which is more natural and not causing problems like older applications.

Natural movement is often limited by real surroundings, as user may need to move in larger area but that's not possible in real setting. In this case application provides procedure of teleportation, where user can change location instantly for 
longer distances by pointing at the new location and pushing joystick forward. When user releases the joystick, application moves him/her to the new location.

Application also provides "step back" function, used go back and turning joystick left or right rotates user in desired direction.

\section{User Interface}

User interface (UI) should consist of selection menus or panels with additional information to user's visual experience.

Since UI will be presented in VR, there are specific rules for text to be easily readable and provide understandable options.

Important text information should be kept in user's field of view (FOW) as it is something users should be aware of in whatever direction they are looking at. Displayed panels should be far enough, not blocking the view, but fitting in. The size of font of every text information should be large enough so users will be able to read it. Aiming at the buttons should not be challenging task to feel selection more natural, achieved by selecting proper size of buttons.

Providing information in more different panels step by step also works better for VR than displaying everything all at once.

Curved menus (Fig. 3) for bigger panels are preferred unlike straight menu panels where content on the sides is in greater distance, which results in unequal feel of presented options from side of user.

Curve angle should not be too high to avoid claustrophobic feeling. This could be done by moving the center of an imaginary circle, where lies curved menu panel, behind user's back.

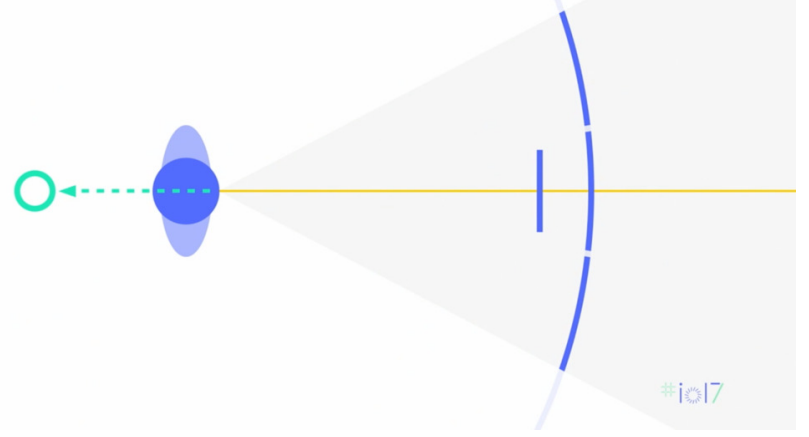

Fig. 3. Layout of curved menu with circle center shifted behind users back [14]

\section{Environment}

Environment needs to provide enough space for users to simulate physical body movement actions and also free space where custom workplaces will be built.

Both indoor and outdoor scenes (Fig. IV-D) can be used for the simulation. Indoor scenes would need correct lightning settings and outdoor scene raised skyline to make scene look infinite. Illusion of no boundaries improves user experience (UX).

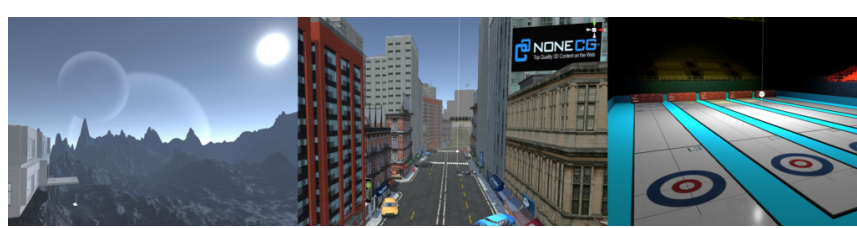

Fig. 4. Outdoor / Indoor scene examples

\section{WORKPLACE BUILDING}

Building of a scene in the application will be a process, where user selects an object from a set of provided 3D models and places these objects to desired location in order to build either a replica of existing workplace or to create and test a new workplace.

Provided items will be sorted into categories to make selection faster. Each item should provide detailed look before selection. Item position in environment will be selected by pointing a controller and pushing button for accept (Fig. 5).

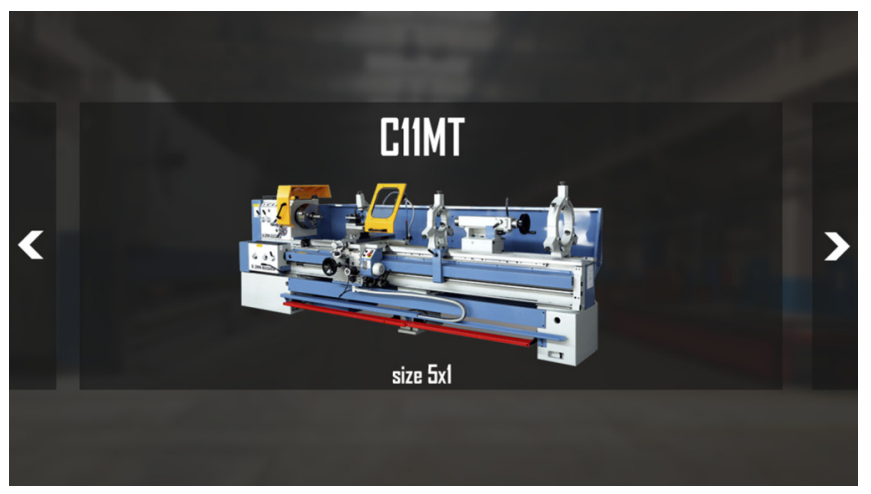

Fig. 5. Proposal of item selection menu

\section{WORK SIMULATION}

Simulation consists of playing recorded body movements of a worker during his/her work.

Users will be able to load film box format (.FBX), which, in addition to polygon and material data of 3D model, also contains animation data. This data will be automatically processed and mapped to 3D engine's animation component.

Loaded animation will be bound to the $3 \mathrm{D}$ model of a human figure used as a mannequin. Playing the animation will move mannequin accordingly.

The applications will also provide a control mechanism for control play speed of an animation based on music or video players (Fig. 6).

\section{A. Limitations}

Setting up limits is important to maintain performance of the application.

This could be done by restricting of number of objects that can be placed in single workplace. The application should also focus on polygon optimization of 3D models. Reducing 


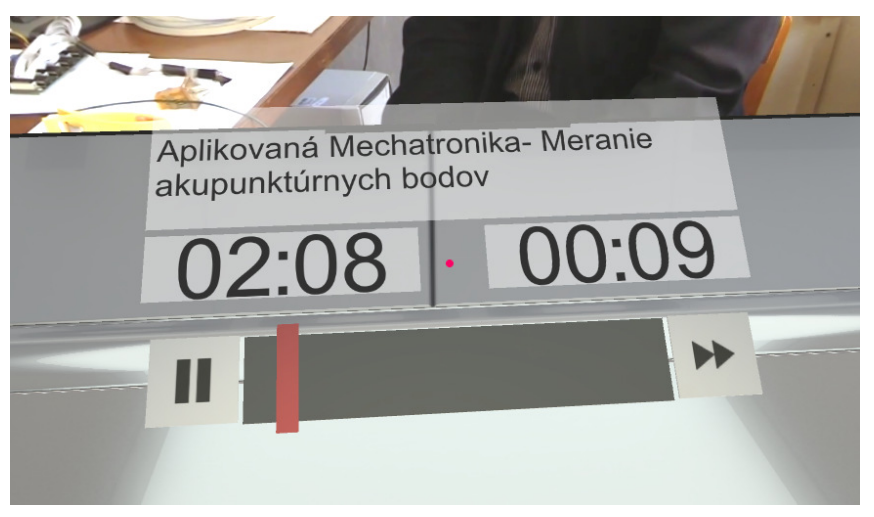

Fig. 6. Video player as an idea for animation player

polygon count in scene will reduce calculation count needed for rendering. Polygon optimization is useful when working with 3D models from CAD. CAD models provides too many details that are not needed in virtual reality visualization (Fig. 7).

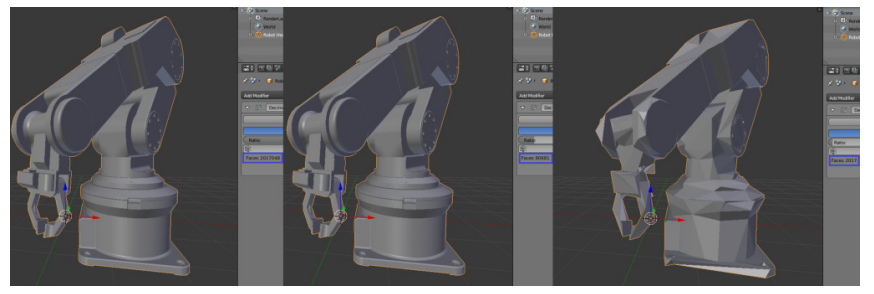

Fig. 7. Example of reducing number of polygons of a 3D model

Another restriction may be setting up a movement area boundary, which defines location, where user can freely move and those places, that are not accessible.

\section{IMPLEMENTATION AND RESULTS}

The vision of application was implemented and built by Unity 3D engine. Unity provides build for a lot of platforms including Windows, Mac, Linux, Android, iOS, etc. WMR headsets are bound to operating system Windows as it is the only operating system supported by these devices.

Unity 3D engine is perfect choice for this type of application. The 3D engine has tools and components created for use with virtual reality. Great advantages of this $3 \mathrm{D}$ engine are low learning curve, easy scripting system and simplicity of engine's GUI.

\section{A. Scenes}

The application itself is divided into two scenes. The first scene provides simple menu with options of loading animations or workplaces while the second scene is used for building and simulation.

Menu-selection scene is a starting point, where user can load animations and open workplaces. This is a small scene where users find themselves inside a room with reflections to optically enlarge surrounding area.

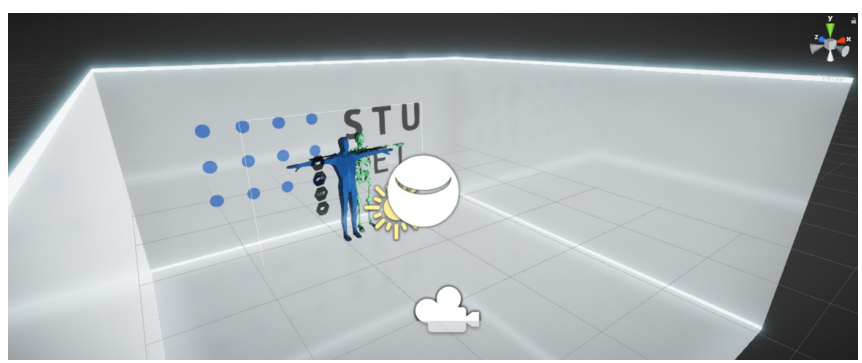

Fig. 8. Main menu scene

Loading of animations is done via system file browser panel, which has similar functions to the traditional Windows file browser. Selection is confirmed by aiming at desired option an clicking the confirm button on virtual reality controllers.

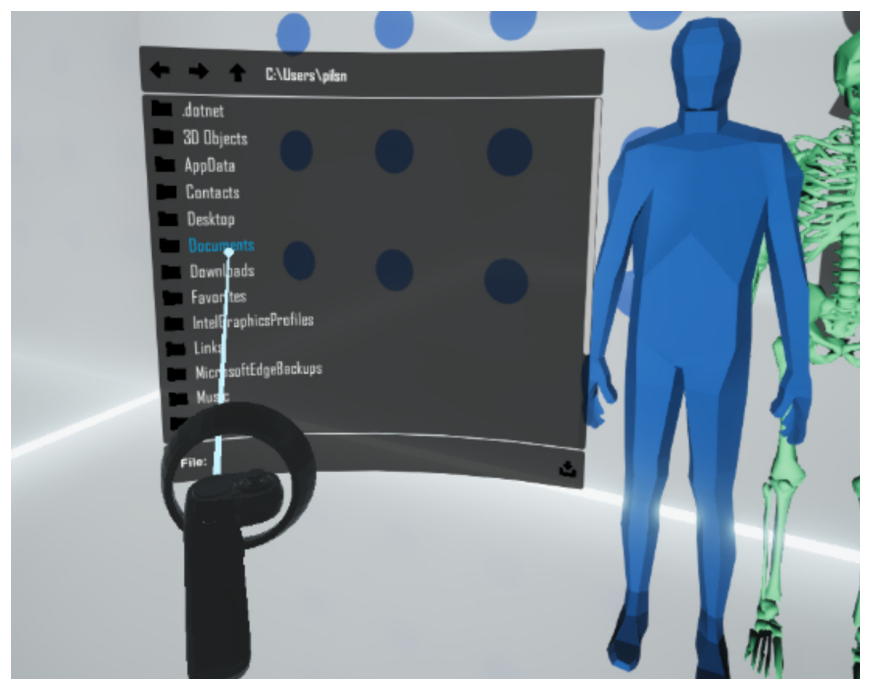

Fig. 9. File browser

Second scene is loaded after animations and workplace are selected. User spawns inside a hangar building. This scene serves for building virtual replicas of actual workplaces. Hangar should provide enough space also for larger workplaces. The door on one of the sides of the building are opened.

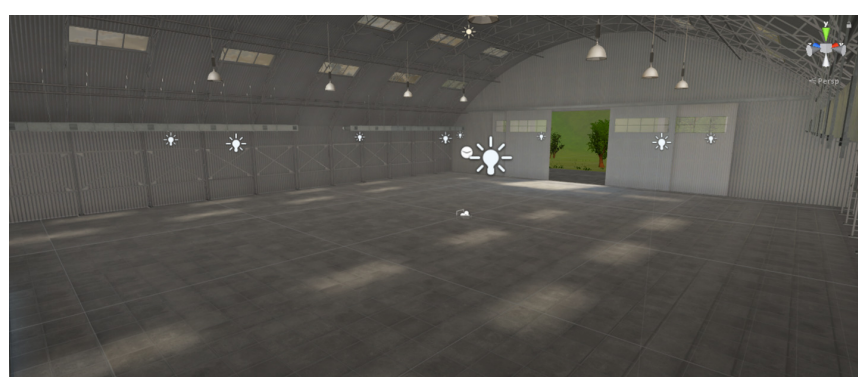

Fig. 10. Simulation scene

Outside area contains grass and trees. Also bird sounds can be heard. Even though outside area is not accessible, it creates a relaxed atmosphere. 


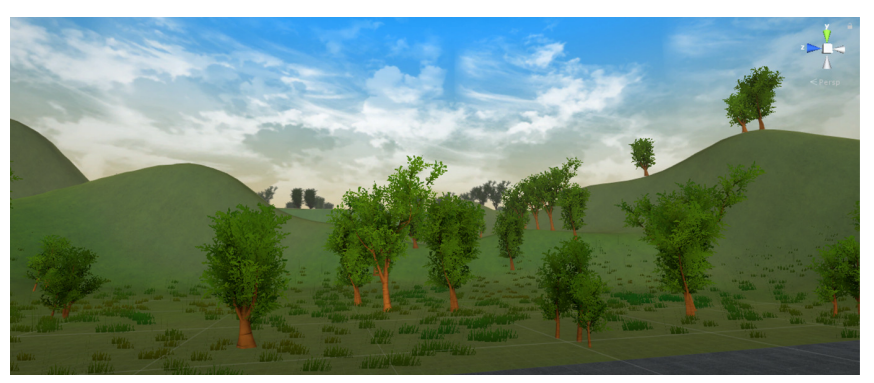

Fig. 11. Outdoor environment of the simulation scene

\section{B. Controllers}

During application runtime, virtual models of controllers are being rendered for users, if they are turned on and paired with computer. Virtual controllers increase amount of interactivity. Selection ray is displayed with a dot on its end which works as a mouse cursor. Aiming and hovering over buttons and objects with scripted actions will result in selecting these components.
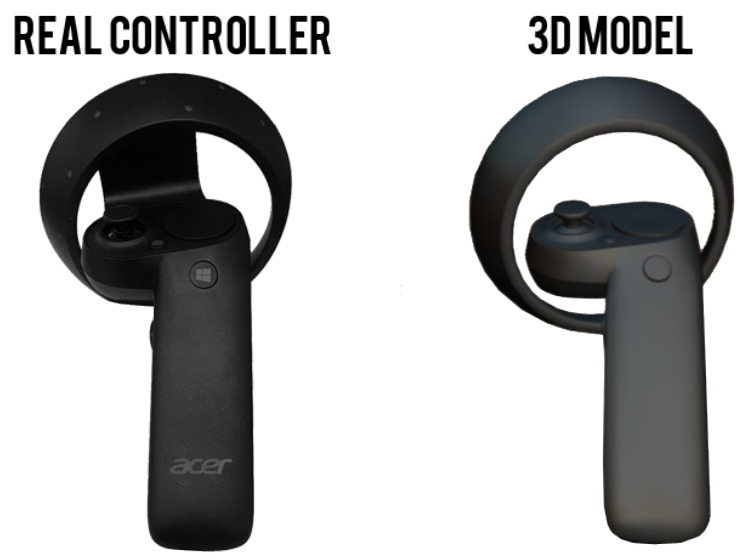

Fig. 12. Real and virtual controller

Movement realization is done exactly as proposed. The application provides four types of movements:

- Real movement projected into application - whatever move user do with headset same change is performed by in-game camera

- Teleport - pushing joystick forward and aiming at the ground, users can teleport to a new location

- Step back - pushing joystick back will result at camera moving back a little

- Turn left/right - turn by 30 degrees by pushing joystick left or right

While requested teleporting by pushing joystick forward, markers to indicate ongoing action are displayed to the user.

\section{Raycasting}

Method of raycasting is used for collision detection. During detection a ray is cast in a certain direction for specified length and first found interception is returned as a collision point.

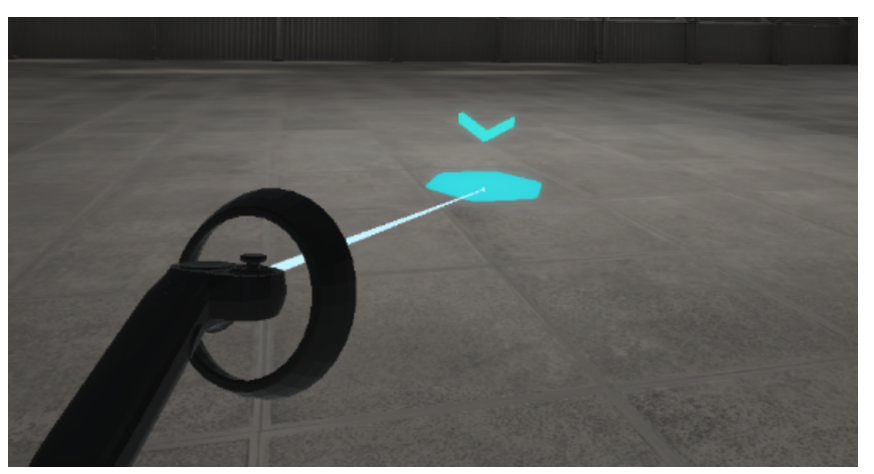

Fig. 13. Teleportation sign

In this case, rays are cast from controllers, checking for editable objects and menu item detections. If confirm button is pressed and user is aiming at such object or menu item at that moment, its action is called in form of $\mathrm{C \#}$ method written in abstract editable object class overridden for specific use of specific object user is currently clicking on.

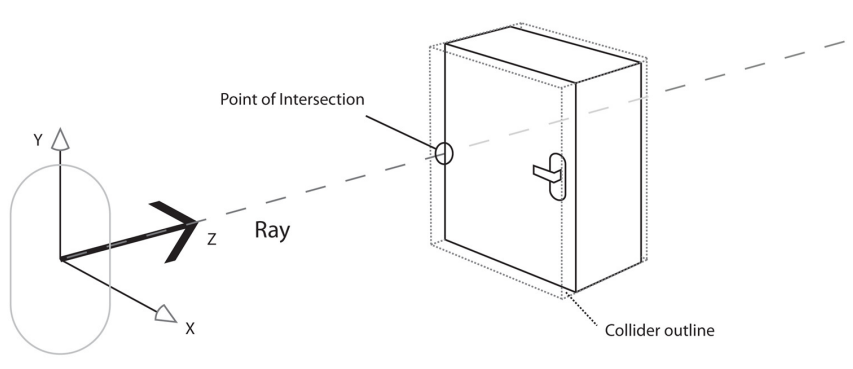

Fig. 14. Raycasting method [15]

As some of the meshes can have too many polygons and complex shapes, colliders are used to simplify these shapes and to receive the collisions. This approach reduces the quantity of calculations needed, although collisions are not always that precise as simplified shape cannot always perfectly align complicated shape.

In Fig. 15, green lines represent edges of a chair collider around its mesh. Colliders at the top of the objects are defining mesh better that those at the base. Reason is that higher parts of collider are more likely to stand in from of other object from user's perspective, which causes blocking rays and detecting object at the front when aiming its direction. When these colliders are better defining its mesh, change-over the objects while moving the controllers is more accurate, representing what user really see. At the bottom areas, while aiming at the ground, there is unlikely that collider could block another object, so those can be simplified so that they don't correspond with actual mesh.

\section{Object Selection And Building}

Building of a workplace is done by selecting an object by object from application menu and placing and positioning the object to fit user needs. Objects provided are categorized. 


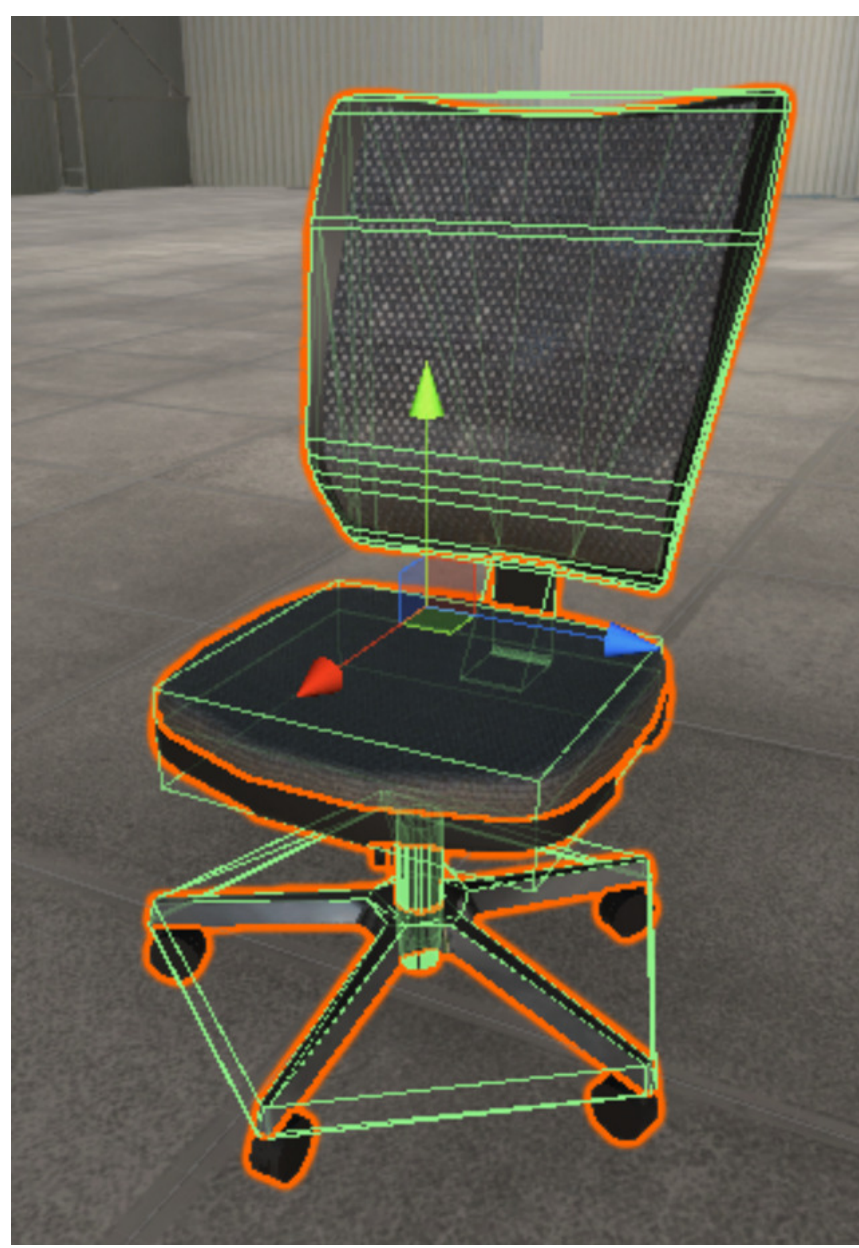

Fig. 15. Object collider example

Menu provides these categories as buttons. Each category contains several optimized objects prepared for use. Items are loaded automatically from application resources based on the folder names. After clicking on category button, objects of selected category are displayed to the user.

Provided categories are:

- Machinery

- Conveyor belts

- Racks

- Tables

- Chairs

- Cabinets

- Electronics

- Other - other objects that do not belong to any of mentioned categories like decoration objects, boxes, walls, etc.

- Primitives - if users cannot find suitable object of those provided by the application, they can replace it with this simple primitive 3D objects as cube or sphere

- Functional - this section contains physical animation player that can be used to control the movement simulation. Category was also prepared for objects like doors or elevators that was not implemented yet

- Mannequin - contains humanoid 3D model

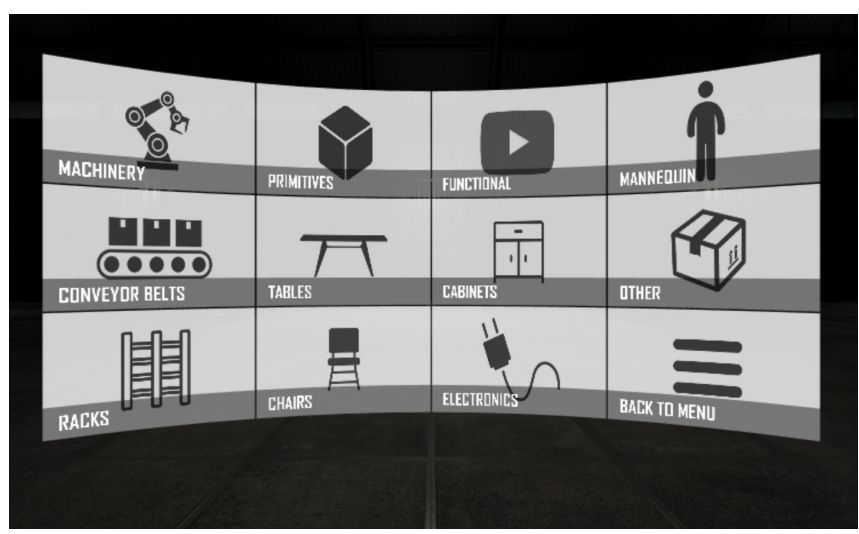

Fig. 16. Build menu

Providing limited number of objects users can use to build their workplaces, it can be hard to create something that looks similar to the real environments. If application contained only very specific $3 \mathrm{D}$ models, it would be impossible task.

Due to this cause, whole specific assembly lines were split into smaller reusable parts that can be used multiple times with different setup (size or colour). Doing this, users create their own specific solution themselves using the principle of modularity (Fig. 17) by combining several objects in many ways.

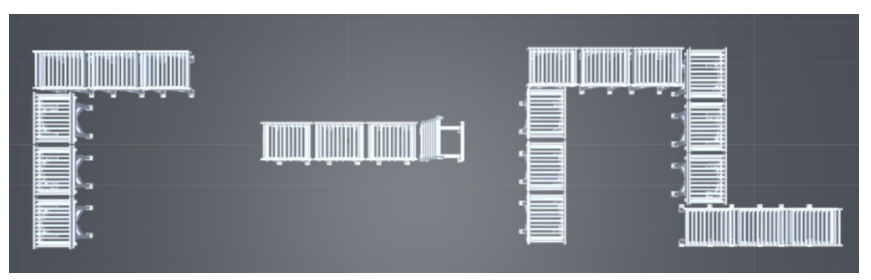

Fig. 17. Modularity principle

\section{E. Object Detail}

When object category in build menu is selected, objects that belong to the category are displayed to the user. There are several objects in each section which could get confusing when showing all at once, especially when also visual of every object has to be displayed. Instead, application presents one object at a time, providing name of the object and detailed look. Arrows on both sides of the object detail can be used to cycle through all objects.

Preview of each object is rendered dynamically. The detail canvas contains a render texture, which can be used to display an image from a camera. Whenever user opens a selection menu, selected item is placed under the whole scene in front of prepared detail camera, where user cannot see it. This camera renders an image, that is projected in the detail canvas of selection menu. When changing the object, old object selected 


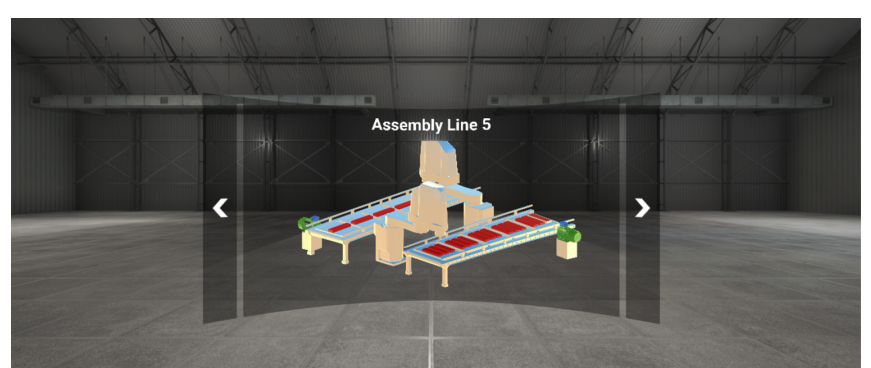

Fig. 18. Selection menu

before is deleted and replaced by the new one just being selected. This will result in change of camera view, which also renders whole new image on the menu canvas.

Detail camera uses orthogonal view, which makes 3D object look more like an image.

\section{Perspective}

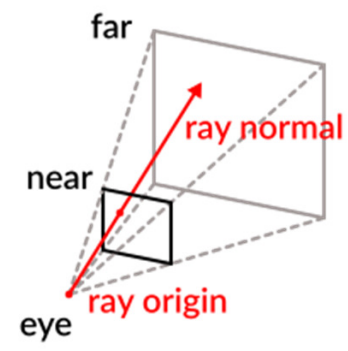

\section{Orthogonal}

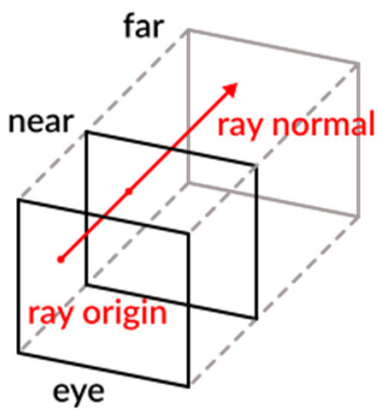

Fig. 19. Orthogonal camera render principle [16]

User confirms the selection by clicking the object image in the canvas, which results in closing the menu and sticking the object to the end of the ray casted from controller, changing its position whenever controller is moved and casted ray collides with the ground. At this moment, object can be rotated moving the joystick right or left. Aiming at desired position and by clicking the submit button, object stops following the ray and becomes solid part of the virtual environment.

\section{F. Object Set-Up Options}

After selecting the object and confirming its position it stays still. But sometimes it is necessary to be able to change object's rotation or position again or delete the object after its building.

All possible modifications of each object are presented by the setup menu. This menu can be accessed by aiming at the built object and pressing the submit button. When aiming at the object, name of the object and the action are automatically displayed to let user know about the action.

Setup menu provides numerous object settings:

- Change rotation - buttons can be used to change the rotation of placed object. Rotation is changed on three euler axes.
- Change scale - rescaling the object on three axes.

- Materials - preview of all mesh materials - clicking the material will open a material menu

- Detail - similar to the selection menu, creates a copy of modified object. All of the modifications are done affecting only this copy and only by clicking the confirm button copy replaces old object in scene. If not confirmed and cancel/back button is pressed, menu closed and object copy is deleted, preserving original object.

- Buttons

- Confirm - confirms the changes

- Move - closes the menu and sticks the object to the end of the ray, user can move object again to its new location

- Delete - deletes object from scene

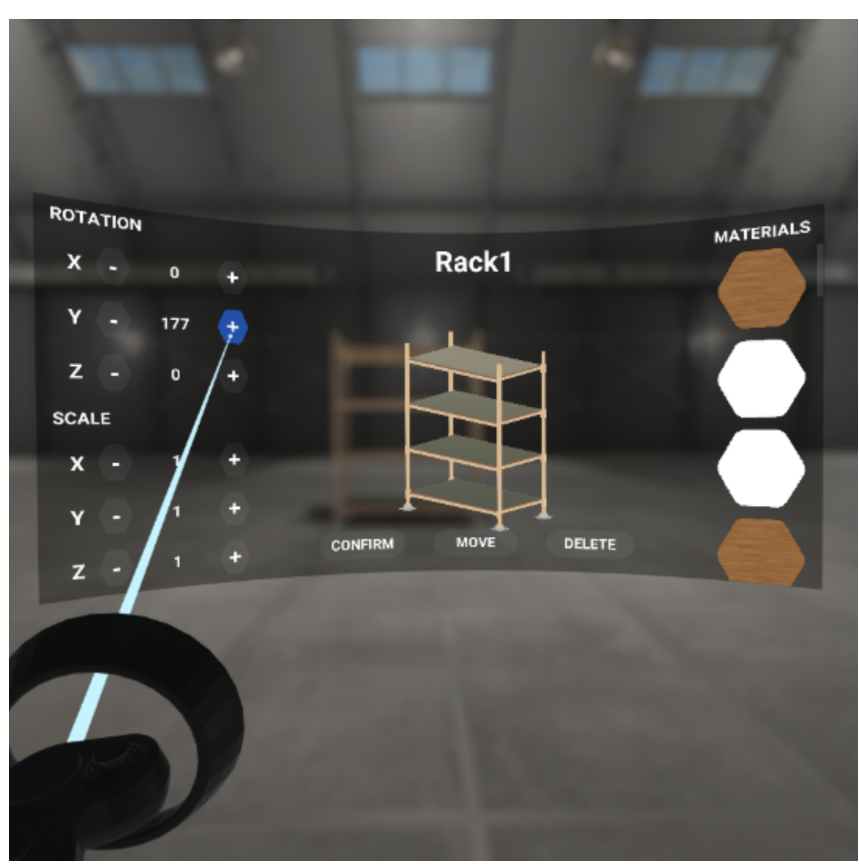

Fig. 20. Object editing menu

Selecting the material from the setup menu opens other menu with all the materials grouped by their type. Clicking on new material will replace the original one. All the materials can be changed. Provided material groups are:

- Simple color

- Fabric

- Glass

- Metal

- Plastic

- Stone

- Wood

Setup menu provides only few options to customize objects, however it can be used to produce totally different object with the identical base 3D model. As an example, here is a cabinet that was rescaled, and materials were changed. 


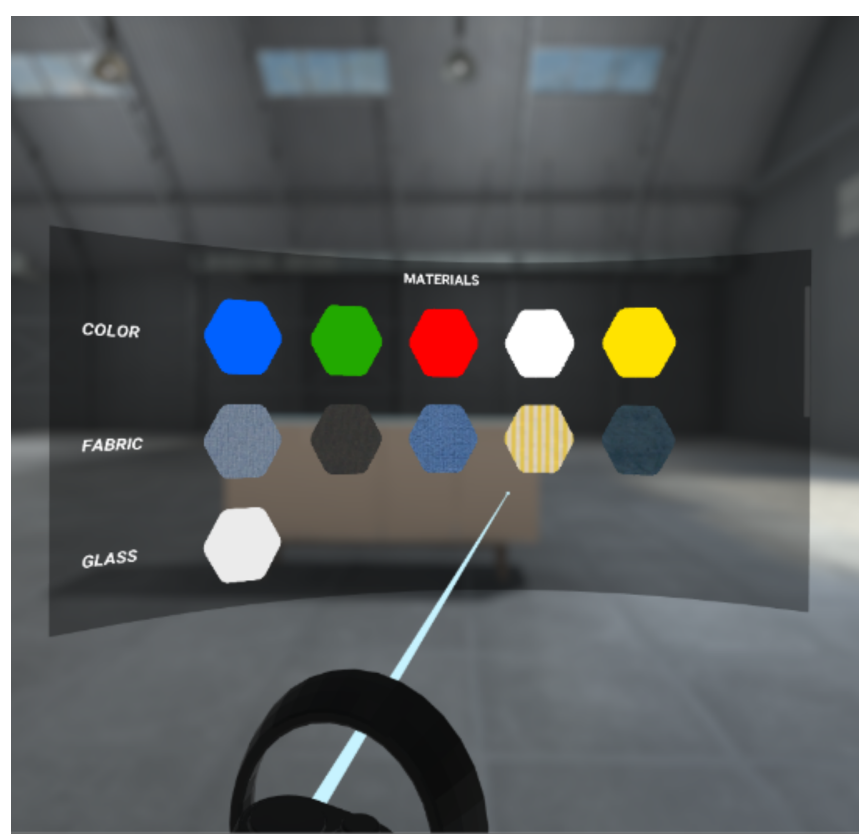

Fig. 21. Material selection menu

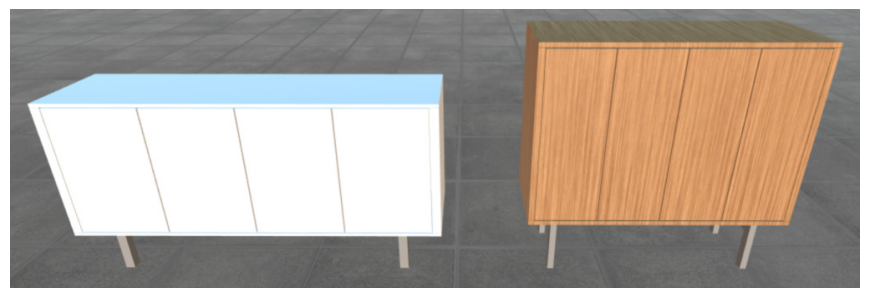

Fig. 22. Example of original and edited object

\section{G. Motion Simulation}

The application provides tools for the simulation which is in this case done by playing recorded motion data as animation bound to the humanoid 3D model. At first, motion data needs to be captured using motion tracking technology. Data have to be stored as .FBX format which can describe also animation data stored in time frames. This file is then loaded through the application using the file browser.

Unity 3D engine does not provide tools for runtime load of an animation or model to the application (except for asset bundles, which is not suitable for this solution, due to the need of the Unity editor to create a bundle), so an external library is used and included to the application.

Asset Import library is an open-source library dedicated for the import of multiple 3D model data formats into the form, it can be easily processed in third-party applications.

With use of mentioned library, animation data is extracted from loaded .FBX file, stored into animation time frames that form whole animation. Each frame has information about positions, rotations and scales of animated object at a specific moment in time. Changing object position and rotation according to this information continuously, frame by frame, will create a visual animation. After animation was loaded, user is informed about animation statistics like total number of frames, framerate (frames per second) and duration of loaded animation. Then users may select an environment in which simulation takes place.

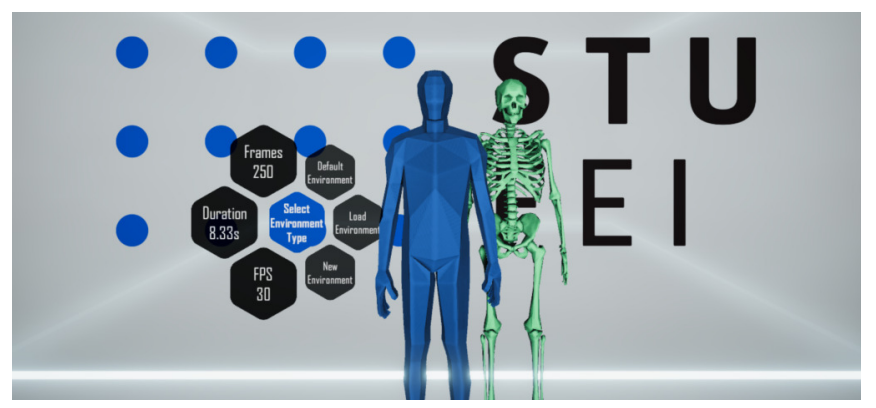

Fig. 23. Animation information after load

In environment, animation is automatically bound to the mannequin - 3D humanoid. To perform the simulation, its necessary to play the animation, which can be done by the controllers as there are keys for play/pause as well as controls for controlling the play speed. In section "functional" of build menu, users can also find physical controller composed of buttons. This controller provides additional information about play speed, animation name, duration and actual time and buttons with the same functionality as described for VR controllers.

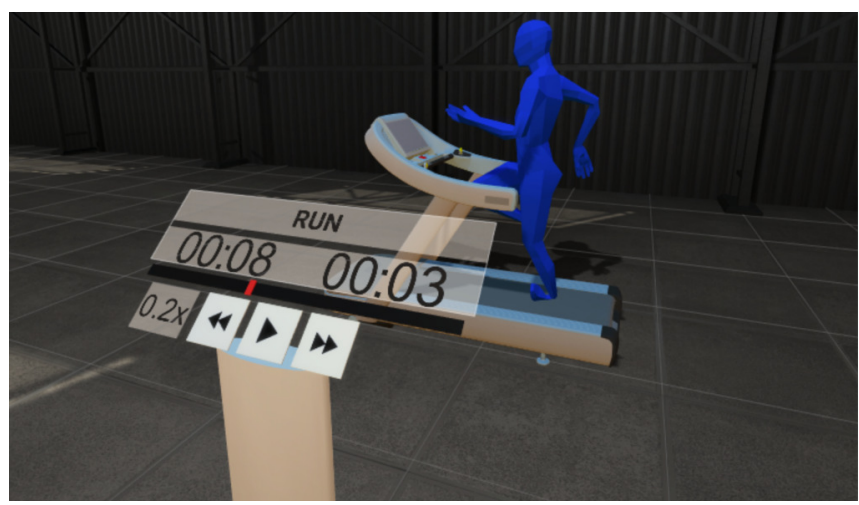

Fig. 24. Motion simulation

\section{H. Results}

Many kinds of environments can be built within the proposed application using / editing provided objects.

\section{CONCLUSION}

The application was developed to help evaluate ergonomics of workplaces with focus on manual repetitive monotonous working activities. One of the main goals was to make the application affordable for small and medium enterprises. The application allows use of affordable motion tracking solutions, which ensures a price drop. 


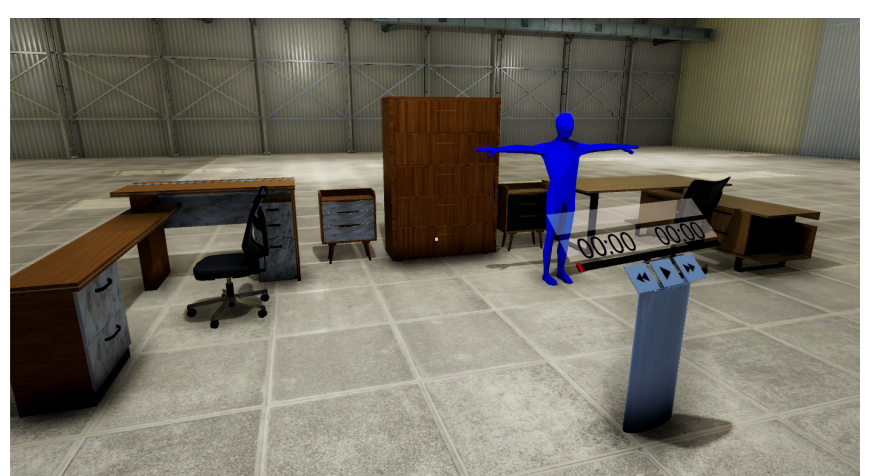

Fig. 25. Office workplace example

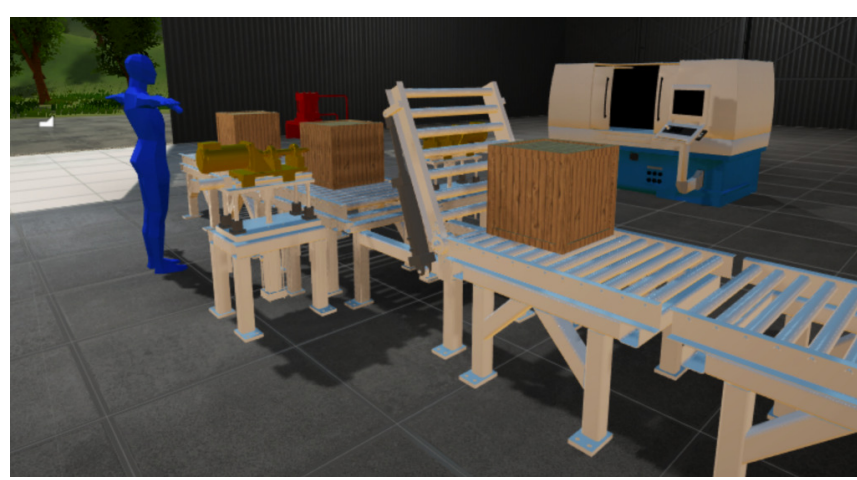

Fig. 26. Industrial workplace example

Main purpose of this application is to provide an alternative original look at the working process, create new points of view using the virtual reality technology and provide tools for managing environment and working process in it.

The application can also be used for workplace adjustments and modifications to fit the ergonomics standards or to change design of evaluated environment to solve its deficiencies. It can also be used for designing entire new environments and in some limited way for training of new employees.

The application is not evaluating ergonomics itself, as it needs specialist in this field to do the evaluation. Automatic machine evaluation of workplaces is one of the improvements we are considering to the future. There is also software like Biomechanics of Bodies, that can automatically evaluate ergonomics of human body based on human joints positions and rotations, counting muscle stress and much more. Using software like this, the application could provide users with evaluated data, directly displaying critical human body parts or exact muscles that suffer the most and marking critical area where unnatural movements occur.

Other improvements can provide more user actions and customization to make it suitable for employee training and augmented reality support could bring evaluation to the real workplace.

\section{ACKNOWLEDGMENT}

This work has been supported by the Cultural and Educational Grant Agency of the Ministry of Education, Science, Research and Sport of the Slovak Republic, KEGA 030STU4/2017 and KEGA 038STU-4/2018, by the Scientific Grant Agency of the Ministry of Education, Science, Research and Sport of the Slovak Republic under the grant VEGA $1 / 0733 / 16$, and by the Young researchers support program, project No. 1328 - KVPRI (Quality Control of Production Processes with Augmented Reality in Industry 4.0) and project No. 1327 - VTOVI (Virtual Training of Production Operators for Industry 4.0).

\section{REFERENCES}

[1] T. Lojka, P. Satala, J. Mocnej, and I. Zolotová, "Web technologies in industry hmi," 09 2015. doi: 10.1109/INES.2015.7329647 pp. 103-106.

[2] J. Filanova, "Application of didactic principles in the use of videoconferencing in e-learning (in slovak)," in Innovation process in e-learning. EKONOM, March 2013. ISBN 978-80-225-3610-3 pp. 1-7.

[3] D. Consulting. (2014) What is ergonomics? [Online]. Available: https://www.ergonomics.com.au/what-is-ergonomics/

[4] J. Krišt'ak. (2017) Ergonomic workplace layout (in slovak). [Online]. Available: https://www.ipaslovakia.sk/sk/ipa-slovnik/ ergonomicke-usporiadanie-pracoviska

[5] S. Hashimura, H. Shimakawa, and Y. Kajiwara, "Automatic assessment of student understanding level using virtual reality," in Proceedings of the 2018 Federated Conference on Computer Science and Information Systems, ser. Annals of Computer Science and Information Systems, M. Ganzha, L. Maciaszek, and M. Paprzycki, Eds., vol. 15. IEEE, 2018. doi: 10.15439/2018F268 pp. 39-45. [Online]. Available: http://dx.doi.org/10.15439/2018F268

[6] A. Lešková, "Ergonomic aspects of workplace design (in slovak)," Transfer inovácií, vol. 7, 2004.

[7] M. Hovanec, "Progressive methods in workplace optimization (in slovak)," Transfer inovácií, vol. 25, 2013.

[8] J. Šsták and J. Čuchranová, "Design of ergonomic construction manufacturing systems for manual assembly (in slovak)," Transfer inovácií, vol. 25, 2013.

[9] K. Procházková, "Ergonomics in vehicles of Škoda auto (in czech)," 2014.

[10] K. Zhang, J. Suo, J. Chen, X. Liu, and L. Gao, "Design and implementation of fire safety education system on campus based on virtual reality technology," in 2017 Federated Conference on Computer Science and Information Systems (FedCSIS). IEEE, 2017. doi: 10.15439/2017F376

[11] J. Majernik, M. Madar, and J. Mojzisova, "Integration of virtual patients in education of veterinary medicine," in 2017 Federated Conference on Computer Science and Information Systems (FedCSIS). IEEE, 2017. doi: $10.15439 / 2017 \mathrm{~F} 134$

[12] S. Paszkiel, "Control based on brain-computer interface technology for video-gaming with virtual reality techniques," Journal of Automation, Mobile Robotics and Intelligent Systems, vol. 10, no. 04, pp. 3-7, 2016. doi: 10.14313/JAMRIS_4-2016/26

[13] H. Kim, N. Hong, M. Kim, S. Yoon, H. Yu, H.-J. Kong, S.-j. Kim, Y. J. Chai, H. Choi, J. Choi, K. E. Lee, S. Kim, and H. Kim, "Ap-

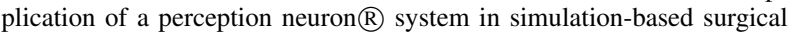
training," Journal of Clinical Medicine, vol. 8, p. 124, 01 2019. doi: $10.3390 / \mathrm{jcm} 8010124$

[14] G. Developers. (2017) Designing screen interfaces for vr (google i/o '17). [Online]. Available: https://www.youtube.com/watch?v= ES9jArHRFHQ

[15] T. Lintrami, Unity 2017 Game Development Essentials, 3rd ed. Packt Publishing, Jan. 2018.

[16] J. Linietsky and A. Manzur. (2018) 3d ray casting from screen. [Online]. Available: http://docs.godotengine.org/en/3.0/tutorials/physics/ ray-casting.html 